\title{
Maltrato entre pares o "bullying". Una visión actual
}

\author{
ALBERTO TRAUTMANN M.,2 \\ 1. Pediatra, Servicio de Pediatría, Hospital Militar. \\ 2. Unidad de Atención Integral del Adolescente, Servicio de Pediatría, Clínica Alemana de Santiago
}

\begin{abstract}
Abuse among partners or "bullying”. Current concepts

An up to date on scholars abuse or "bullying" is presented. Its significance, role, characteristics, consequences and epidemiology are explained. Schools interventions and their results are reviewed and an analysis of health team role is performed. A family and patient counseling is proposed. There is a special emphasis on the witness or bystander role in schools and health interventions performed.

(Key words: Abuse, partners, bullying, witness, adolescent).

Rev Chil Pediatr 2008; 79 (1): 13-20
\end{abstract}

\section{RESUMEN}

Se presenta una actualización sobre el acoso o maltrato escolar entre pares o "bullying". Se explica su significado, el rol y características de sus actores y sus consecuencias. Se menciona su epidemiología y los factores condicionantes que influyen en su presencia. Se revisan las intervenciones realizadas en los colegios y sus resultados, y se analiza el rol de los prestadores de salud. Se proponen indicaciones de consejería a la familia, con algunos datos útiles para usarlos en la consulta con los pacientes. Se hace especial énfasis en el rol del testigo (bystander) o espectador tanto en las intervenciones escolares realizadas, como que éste sea foco de atención en la consulta de los profesionales.

(Palabras clave: Maltrato, pares, matonaje, bullying, testigo, adolescente).

Rev Chil Pediatr 2008; 79 (1): 13-20

\section{Introducción}

El maltrato entre pares o "bullying” en inglés es una realidad que ha existido en los colegios o escuelas desde siempre y se ha considerado un proceso normal dentro de una cultura del silencio que ayuda a su perpetuación. En Europa, Estados Unidos, Canadá y Australia es un tema vigente desde hace décadas. En Chile ha adquirido notoriedad gracias a los medios de comunicación e internet, por la difusión de las serias consecuencias para sus 
participantes, y éstos, que en un principio se consideraba que eran la víctima y el victimario, hoy se entiende que además son partícipes de la dinámica los espectadores, testigos o "bystanders" directos que presencian el hecho, $\mathrm{y}$ los indirectos, que son el personal, las autoridades del colegio, la familia y la sociedad entera. Por lo tanto, la solución no está enfocada a una o dos personas en particular, sino que debe involucrar a toda la comunidad. Dentro de la comunidad está el personal de salud y específicamente el pediatra quien puede detectar esta realidad oculta y orientar en las acciones a seguir.

\section{Matonaje o bullying, en qué consiste}

Es un comportamiento agresivo que implica tres aspectos: desbalance de poder, que se ejerce en forma intimidatoria al más débil, por lo tanto, escogido y no al azar, con la intención premeditada de causar daño, y que es repetido en el tiempo.

El tipo de bullying puede ser directo, ya sea físico o verbal, o de gestos no verbales. Puede ser indirecto o relacional (daño a una relación social), mediante la exclusión social, e1 esparcir rumores, o hacer que sea otro el que intimide a la víctima ${ }^{1}$.

Últimamente se ha agregado el cyberbullying, que se refiere al matonaje que se realiza bajo anonimato por internet, usando blogs, correo electrónico, chat y teléfonos celulares, enviando mensajes intimidatorios o insultantes.

\section{Visión del Problema}

Prevalencia: depende de la definición de esta dinámica y de la frecuencia de los episodios. Si se considera que un episodio de "bullying" basta para hacer diagnóstico, el bullying aumenta en la estadística. En un estudio de Nansel en 2004, en una muestra de 113200 estudiantes de 25 países, se observó que desde el 9\% en Suecia, hasta un 54\% de los escolares en Lituania, estaba involucrado en episodios de "bullying”. Como víctimas, el rango va desde un 5\% en Suecia hasta un 20\% de los estudiantes en Lituania, con un promedio en todos los países de un 11\%. Como agresores, se comunicó desde un 3\% en Suecia hasta un $20 \%$ en Dinamarca, con un promedio de $10 \%$.
El papel dual de agresor y víctima varió desde un $1 \%$ en Suecia hasta un $20 \%$ en Lituania, con un promedio en los países de $6 \%{ }^{2}$. En Chile, la participación de los estudiantes en conductas de matonaje oscilaría entre un 35 a $55 \%{ }^{3}$.

Edades: de acuerdo a Olweus, en $4^{\circ}$ básico el problema sería el doble que en $8^{\circ}$ básico. Nansel encontró algo similar al comparar alumnos de $6^{\circ}$ básico, en que el $25 \%$ habían sido victimizados, con los de $10^{\circ}$ grado, en que esa dinámica se verificaba en menos de un $10 \%{ }^{4}$.

Género: los niños varones victimizan más que las niñas y utilizan más la agresión física y verbal. Las niñas usan la agresión indirecta relacional, esparciendo rumores o realizando exclusión social ${ }^{1}$.

Lugar del maltrato: el patio del colegio o lugar sin supervisión de adulto es donde ocurren las agresiones físicas. En clase, con o sin profesor presente, ocurren las agresiones verbales $^{1}$.

\section{Características personales de los actores y consecuencias}

Las víctimas: son percibidas como inseguras, sensitivas, poco asertivas, físicamente más débiles, con pocas habilidades sociales y con pocos amigos. En general, buenos alumnos ${ }^{1}$. Como consecuencia del bullying, presentan ansiedad, depresión, deseo de no ir a clases con ausentismo escolar ${ }^{5}$ y deterioro en el rendimiento ${ }^{6}$. Presentan más problemas de salud somática, dos a cuatro veces más que sus pares no victimizados ${ }^{7}$. Si la victimización se prolonga, puede aparecer ideación suicida ${ }^{8}$. Ello es favorecido por el poder en aumento del agresor y el desamparo que siente la víctima, con la creencia de ser merecedora de lo que le ocurre, produciéndose un círculo vicioso, lo que hace que sea una dinámica difícil de revertir ${ }^{9}$. En la adultez, hay mayor desajuste psicosocial ${ }^{10}$.

Los agresores o bullies: son físicamente más fuertes que sus pares, dominantes, impulsivos, no siguen reglas, baja tolerancia a la frustración, desafiantes ante la autoridad, buena autoestima, tienen actitud positiva hacia la violencia, esperan crear conflictos donde no los hay, no empatizan con el dolor de la víctima, ni se arrepienten de sus actos. Como consecuencia de su conducta, adquieren un patrón para 
relacionarse con sus pares, consiguiendo sus objetivos con éxito, aumentando su status dentro del grupo que los refuerza. Al persistir, caen en otros desajustes sociales como vandalismo, mal rendimiento académico, uso de alcohol, porte de armas, robos, y de acuerdo a Olweus, procesos en la justicia por conducta criminal en un $40 \%$ a la edad de 24 años ${ }^{11,2}$.

Los bully-víctimas: tienden a ser hiperactivos, con dificultad para concentrarse. Son impulsivos, de tal modo que devuelven el ataque. Cuando son atacados, muchos estudiantes están involucrados como agresores, a veces, la clase entera. Ellos tienden a maltratar a niños menores o más débiles que ellos. Son los que han acusado más problemas de salud, más que las víctimas. Han tenido más problemas académicos, más que los agresores, tienen problemas de relación con sus pares y mayor uso de tabaco y alcohol ${ }^{1,2}$.

Los testigos, espectadores o bystanders: son la audiencia del agresor, entre el 60 y $70 \%$ del universo restante. El agresor se ve estimulado o inhibido por ellos, es por ello que hay programas de prevención que tratan que los testigos tengan un rol preventivo ${ }^{12}$. En un estudio, el 30\% de los testigos "intentó ayudar a la víctima”, mientras que el 70\% no intentó intervenir. Desglosada esta cifra, el $40 \%$ no hizo nada porque "no era de su incumbencia", y el $30 \%$ no ayudó aunque "sintieron que deberían hacerlo”, probablemente por temor a ser víctimas $^{13}$. Hay una intención que no se traduce en conducta, lo que podría ser modificado.

Hay un segundo universo de testigos que son los profesores y personal del colegio. Según la mayoría de los alumnos, los profesores no intervendrían siempre en situaciones de victimización, y sólo un tercio de los alumnos cree que a los profesores les interesa poner fin al "bullying"14.

Las consecuencias para los testigos son el valorar como respetable la agresión, el desensibilizarse ante el sufrimiento de otras personas y el reforzar el individualismo ${ }^{1}$.

\section{Factores del entorno condicionantes}

Se ha visto mayor asociación con acoso escolar entre pares, el que haya padres distantes , poco cálidos, familias poco cohesionadas o padres sobreprotectores, castigos inconsistentes, castigos físicos, padres violentos, victimización entre hermanos, y un padre que haya tenido historia de acoso $0^{1,15,16}$.

En las redes de grupos de pares en que hay más conductas agresivas, hay mayor asociación con maltrato entre pares ${ }^{16}$. Los testigos, según la actitud que tengan, pueden tener un rol fomentador o inhibidor del maltrato ${ }^{12}$.

\section{Intervenciones y sus resultados para prevenir el maltrato entre pares}

Intervenciones curriculares: diseñadas para promover una actitud anti acoso en la sala de clases y ayudar a los niños a desarrollar habilidades para resolver conflictos. Incluyen videos, grupos de discusión por semanas. Se incentiva la toma de conciencia del bullying entre los estudiantes y se promueve la adquisición de valores pro-sociales, como la tolerancia por las diferencias, cambio de actitud de los estudiantes, con cambios en las normas del grupo. Se promueve la asertividad para persuadir al agresor y la actitud activa de los testigos o espectadores ${ }^{17}$. Son intervenciones más atractivas, porque requieren menos recursos, personal y esfuerzo. Sin embargo, no han mejorado el problema, y los que menos se han beneficiado son los niños menores. El fracaso se explica porque el acoso escolar es un proceso sistémico y la teoría apoya que es un fenómeno sociocultural. Si acaso el bullying es un proceso sistémico grupal, que involucra a agresores, víctimas, pares, adultos, padres, ambiente escolar y ambiente del hogar, es improbable que una intervención en un solo nivel tenga algún impacto $^{18,19}$.

Intervenciones de entrenamiento de habilidades sociales en forma individual: basadas en cambios cognitivos y de comportamiento. Los mejores resultados se han obtenido de los niños más pequeños y más en las víctimas pero no en los agresores. Claramente, no han mejorado el problema del acoso escolar ${ }^{20}$.

Intervenciones integrales como una totalidad ("whole school intervention"): son de tipo multidisciplinario y se realiza en distintos niveles en la organización del colegio, pudiendo incluir a las anteriores. Este tipo de intervención asume el "bullying" como un problema 
sistémico que reclama solución a distintos niveles y no sólo a una actividad en clase. Busca influir e intervenir con distintas actividades en todo el ambiente escolar e involucrar a individuos, víctimas, agresores, grupos de pares, salas de clases, profesores, administración, familias en general y familias de víctimas y agresores. El mayor éxito de este tipo de enfoque sugiere que hay factores externos al individuo y que tienen que ver con procesos de interacciones sociales, sin embargo, hay barreras que limitan su efectividad. La implementación varía en forma significativa. El programa integral de Olweus aplicado en Bergen, Noruega, y que fue exitoso, en otras escuelas o países fue de nulo efecto e incluso con un efecto negativo. El éxito dependería de la duración del programa, del compromiso y entrenamiento de los profesores y autoridades del colegio, de la replicación e instrucciones adecuadas en el detalle ${ }^{20}$.

\section{Otro tipo de intervenciones}

Este tipo de intervenciones, como la tutoría por un adulto o por pares, o la intervención sobre el testigo o espectador, no deberían entenderse como acciones aisladas, sino como parte de las intervenciones multidisciplinarias.

Tutoría o "mentoring”: Un estudio de King et al 2002 investigó los efectos de un programa de tutoría para niños “en riesgo”. Los niños con tutores tuvieron significativamente menos reporte de bullying, peleas físicas y sentimientos de depresión en los últimos 30 días. Estos vínculos proveen modelos para ayudar a los niños a aprender habilidades y lenguaje para resolver problemas $^{20}$.

Intervención sobre el testigo o espectador: están dentro de los programas integrales en la toma de conciencia, y en el hacer del colegio un lugar seguro. Hay pocas acciones específicas sobre el espectador, más bien hay investigación para entender por qué actúa en la forma que lo hace. El rol del espectador no es pasivo, sino activo, porque participa en un proceso, no existiendo una díada, sino una tríada en la cual el testigo favorece o disminuye el acoso ${ }^{12}$. Según Rigby et $\mathrm{al}^{21}$, las intenciones de acción en la dinámica son: 1) Apoyo a la víctima en forma directa, que sería por una razón moral, por ponerse en el lugar del otro, por preocupación por los demás, por reciprocidad. 2) Ayuda a la víctima en forma indirecta, lo que sería comunicando la situación a un profesor, ya que eso traería consecuencias tangibles al agresor, como un castigo, existiendo sin embargo el temor a la represalia. Si se tratara de un acoso sexual, no habría temor en decirlo a un profesor. 3) El ignorar el episodio, cuyas razones serían, "porque no es de mi incumbencia”, por temor a las consecuencias, porque la víctima "tiene la culpa”, porque "no sirve inmiscuirse", porque el "espectáculo es bueno y ver peleas es entretenido”. 4) Apoyo al agresor, que se daría porque es la opción más segura, por admiración al agresor, ó porque la víctima “lo merece”. Tanto en varones y mujeres, la intención apoyar o no a la víctima eran anticipadas por la actitud hacia las víctimas (ayuda a la víctima, el ignorar el episodio o apoyar al agresor) y con gran fuerza, las expectativas de los amigos. La presión de los pares es entonces un gran factor. Las expectativas de profesores o padres aparecían como no importantes. La actitud de los testigos no cambiará simplemente porque profesores o padres se los digan. Sin embargo, es importante valorar que, una vez que un niño ha actuado como testigo que ayuda a la víctima, es más probable que lo siga haciendo. Para motivar a los testigos, Rigby da algunas sugerencias que consisten en mostrar en clases un video o cuadros que muestren a testigos mirando a un niño que está siendo agredido y discutir qué es lo que ellos sienten, qué harían y por qué, con los mejores resultados obtenidos en alumnos de enseñanza básica, y menos en educación media ${ }^{21}$.

\section{Papel de los Prestadores de Salud}

Los profesionales que realizan el control de salud preventivo desde la edad de lactante, pueden fomentar un hogar en que los padres sean modelo de consideración, respeto, cariño y afecto para su familia y para personas que no pertenezcan a ella. Que se le enseñe al niño a actuar socialmente, a actuar por razones objetivas y no por miedo a la represalia o al ridículo social, a tolerar la frustración, la diversidad, a manejar la rabia y el stress, a tener límites ${ }^{22,23}$.

Los prestadores de salud deben preguntar sobre esta realidad y el papel que juegan los 
jóvenes en la tríada, sean víctimas, agresores o testigos. En el caso que pesquisen a víctima o agresor, realizar evaluación de comorbilidad psiquiátrica (trastorno de ansiedad, trastorno depresivo, trastorno de conducta) y orientar o dar consejería a la familia ${ }^{24}$.

En el Apéndice 1, tomado y modificado de Olweus, $1993^{1}$, se enumeran algunos signos de victimización que el pediatra pudiera pesquisar. En los Apéndices 2 y 3 hay preguntas que se pueden hacer tanto al niño como a sus padres para pesquisar el fenómeno, tomadas de Glew, Rivara, Feudtner, $2000^{24}$.

Si no se pesquisa a víctima o agresor, obligadamente se tendrá a un testigo o espectador. Es el momento de hacer educación y prevención: se le podría preguntar al paciente por ejemplo, “ $\dot{¿}$ Te ha tocado ver que alguien moleste a otro? ¿En qué ha consistido? ¿Qué has sentido tú en ese momento?” “¿Qué crees tú que siente el que es molestado?” “¿Te parece que es correcto eso que pasa?" "Es difícil muchas veces hacer algo; a ti, ¿te han dado ganas de hacer algo? ¿Lo has hecho? ¿Cuál ha sido el impedimento? ¿Y qué te parece?’. Que el testigo sepa que ayudar a la víctima no es una pelea física. Una forma de ayudar a la víctima es brindándole amistad, ya que los agresores atacan a los que no tienen amigos. Hay que ofrecer la posibilidad al testigo que revise el tipo de amigos que pudiera tener, abrirlo a la diversidad. Que comunique la situación a un adulto. Que eso no es acusar. Es importante decirle que está comprobado que las personas que son víctimas y también agresoras, tienen serias consecuencias psicológicas y sociales, y que su actitud ayudará a las personas ahora y a prevenir en ellos a futuro problemas psicológicos. Indicarles que el rol que cumple es dar o quitar poder al agresor por el solo hecho de estar ahí presente, validando o no lo que ahí sucede. En la el apéndice 4 se propone acciones concretas que a los testigos se les puede sugerir que realicen.

\section{Consejería a la Familia}

A la familia de la víctima hay que explicarle el fenómeno, en qué consiste, cuáles son los actores, cuál es la dinámica que se da, la cultura del silencio que impera, la ignorancia que hay del fenómeno o del hecho específico en los colegios, y la tendencia a la perpetuación de esta interacción en la cual esté el hijo ${ }^{24}$. Por ello, no hay que dejar que haya una evolución espontánea, sino actuar ahora y no después.

Muy importante es no actuar en forma individual y precipitada. Los padres deben recopilar la información exacta de qué ocurre, cuándo, cómo, quiénes (con nombres), hace cuánto tiempo y con qué frecuencia. Los datos vagos no sirven. Y con esa información tomar contacto con la autoridad del colegio. Puede que el colegio tenga una política integral anti acoso escolar. Si no la tuviera, hay que procurar que la autoridad convoque a las personas involucradas y sus padres por separado. La solución es a través de la autoridad del colegio y no con los padres del agresor o con el agresor mismo.

En lo inmediato, a la víctima debe contenérsela, decirle que no tiene culpa de lo que le pasa y el colegio debe asegurarle que esto no seguirá ocurriendo y verificar de que ello ocurra. Hay que hacerle ver que el aparecer inseguro invita a que haya maltrato, de forma que es bueno proyectar seguridad en sí mismo, entre otras acciones, educando una actitud asertiva en lenguaje verbal y no verbal y practicándolo con sus padres ${ }^{25}$. Hay actividades que ayudan a ello, como los talleres de teatro, actividad física y aumentar suficiencia y fortaleza física. Que no ande solo, que haga nuevos amigos, $y$ para facilitar ello, que se incorpore a alguna actividad extracurricular como las mencionadas. No se recomienda que la víctima responda con agresión física. En la referencia 23 diferentes autores muestran una recopilación de técnicas, acciones y recursos a las que se puede recurrir para sugerir a la víctima. Se aconseja evaluación psiquiátrica por presencia eventual de comorbilidades ${ }^{24}$.

$\mathrm{Al}$ agresor la autoridad del colegio tiene que decirle que lo que hace no es correcto, que no va a ser tolerado y que habrá consecuencias si continúa. Se requiere involucrar a los padres. Hay que tratar de saber por qué lo hace, en una conversación, ya que puede ser por imitación de modelos, por presión de pares, por reacción ante situación personal o porque no tiene claro que no es correcto. También es recomendable en estos casos una evaluación psiquiátrica. 


\section{Conclusiones}

El maltrato entre pares escolares, aunque sea común, no es aceptable. El maltrato entre adultos tampoco, pero hay legislación, y los niños carecen de legislación protectora. El acoso trae nefastas consecuencias para víctimas, victimarios y testigos. No debe ser entendido como un problema entre dos personas, sino en una totalidad, en la cual está la tríada que incorpora a los testigos, espectadores o "bystanders”. Y éstos no se limitan a los que presencian directamente el episodio, involucra a la comunidad entera con sus valores. Son los testigos los que dan poder al intimidador. De tal modo, que el abordaje de este problema debe hacerse desde un punto de vista de totalidad, sistémico y multidisciplinario, involucrando al colegio con los subsistemas (administrativos, profesores, alumnos, familias, ambiente del colegio, sociedad, mundo de los adultos). Éstos pueden ser foco de intervenciones.

Los prestadores de salud y en especial los pediatras forman parte de ese sistema y su rol es la pesquisa de víctimas, agresores, educación con sensibilización de los testigos, consejería a los padres e influir en la legislación.

\section{Apéndice 1}

\section{Signos de victimización (Olweus 1993, modificado)}

- El niño o joven regresa del colegio con ropa, libros pertenencias dañadas o sin ellas

- Presencia de heridas, equímosis, cortes inexplicados

- Tiene pocos o ningún amigo

- Se le ve preocupado de ir al colegio

- Evita cierta gente, situaciones, lugares

- Ha perdido interés en el trabajo escolar

- Presenta cefaleas, dolores abdominales o síntomas vagos

- Se queda en casa porque está "enfermo"

- Visita frecuentemente la enfermería del colegio por síntomas y así regresar a casa

- Tiene dificultad para dormir y/o tiene pesadillas

- Se ve triste, deprimido

- Está silencioso, sensible y pasivo
- Estigmas: obesidad, dislalia, espamofemia, discapacidad, trastornos de aprendizaje

\section{Apéndice 2}

\section{Preguntas para investigar el bullying en} los niños (Glew, Rivara, Feudtner, 2000, modificado)

- ¿Qué haces a la hora del recreo, la hora de almuerzo? ¿Juegas con otros o solo? ¿Por qué?

- ¿Hay alguien en tu clase que no te guste?

- ¿Te ha tocado ver que alguien moleste a otro? ¿Te ha pasado algo parecido?

- ¿Con qué tipo de cosas te han molestado?

- ¿Lo han hecho por tu enfermedad/ discapacida/desventaja?

- ¿Lo han hecho porque no te gusta hacer las cosas que ellos hacen? ¿qué cosas hacen ellos? Y mientras, qué haces tú?

- ¿Hace cuánto tiempo está pasando esto?

- ¿Alguna vez se lo has dicho a un profesor o algún adulto esto que pasa? ¿Y qué ha sucedido?

- ¿Te has cambiado alguna vez de colegio porque tenías problemas con otros estudiantes?

\section{Apéndice 3}

\section{Preguntas a los padres para investigar} bullying (Glew, Rivara, Feudtner, 2000, modificado)

- ¿Le ha mencionado el profesor que su hijo pasa la mayor parte del tiempo solo?

- ¿Va su hijo muy frecuentemente a la enfermería del colegio?

- ¿Con qué frecuencia la llaman del colegio porque su hijo se siente mal?

- ¿Con qué frecuencia su hijo amanece con dolores de estómago/cabeza/.... ?

- ¿Le ha dicho su hijo que otros niños lo molestan?

\section{Apéndice 4}

\section{Sugerencias para los testigos o} espectadores

- No reírse junto con el agresor en el acto intimidatorio. 
- Cambiar de escena al agresor: ¡Hey! Te necesitamos en el partido de fútbol.

- Cambiar de escena a la víctima: ¡(nombre de la víctima)! El profesor $\mathrm{X}$ necesita hablar contigo inmediatamente.

- Comunicarlo a un adulto, con información de cuándo y dónde ocurre.

- Quitarle la audiencia al agresor. Irse y animar a otros a hacerlo.

- Parar al agresor de manera firme, involucrando al resto, usando frases como “No queremos esto, ¡córtala!” (si se siente seguro el hacerlo)

- Brindar amistad pública a la víctima.

- Hacerle presente a la víctima posteriormente que no está de acuerdo con lo que sucede.

- Convencer a la víctima que converse la situación con un adulto.

- Invitar a la víctima a entretenciones grupales.

- No participar en rumores ni decir afirmaciones que menoscaben la dignidad de las personas.

- Enrolarse en actividades anti acoso escolar u otras de servicio a los demás, ser tutor de niños menores.

- Ser modelo positivo e integrador, incorporando la diversidad.

- Referise en términos positivos a la autoridad.

- No usar la violencia física con el agresor.

\section{Referencias}

1.- Olweus D: Bullying at school: What we know and what we can do. Blackwell Publishing, 1993.

2.- Nansel TR, Craig W, Overpeck MD, Saluda G, Ruan J: Cross-national consistency in the relationship between bullying behaviors and psychosocial adjustment. Archives of Pediatrics and Adolescent Medicine 2004; 158: 730-6.

3.- Lecannelier F: Un proyecto bullying para la detección e intervención de la violencia escolar entre pare en Chile. Memorias de las XII Jornadas de Investigación y Primer Encuentro de Investigadores del MERCOSUR, Buenos Aires, Argentina 2005.

4.- Nansel TR, Overpeck M, Pilla RS, Ruan WJ, SimonaMorton B, Scheidt P: Bullying behavior among US youth: Prevalence and association with psychosocial adjustment. Journal of American Medical Association. 2001; 285: 2094-100.

5.- Kochenderfer BJ, Ladd GW: Peer victimization: Cause or consequence of school maladjustment? Child Development 1996; 67: 1305-17.

6.- Eisenberg ME, Neumark-Sztainer D, Perry C: Peer harassment, school connectedness and school success. Journal of School Health 2003; 73: 311-6.

7.- Fekkes M, Pijpers F, Fredriks AM, Vogels T, VerlooveVanhorick SP: Do bullied children get ill, or do ill children get bullied? A prospective cohort study on the relationship between bullying and health related symptoms. Pediatrics 2006; 117 (5): 1568-74.

8.- Rigby K: Bullying in schools: And what to do about it. Bristol, PA; Jessica Kingsley Publishers. 1996.

9.- Avilés Martínez JM: La intimidación y el maltrato en los centros escolares. Revista Lan Osauna N²2, 2002.

10.- Olweus D: Victimization by peers: Antecedents and long term outcomes. En Rubin KH, Asendorf JB, (ed.) Social withdrawal, inhibition and shyness in children. Hillsday, NJ: Erlbaum, 1993b: 315-341.

11.- Olweus D: Bullying among school children: Intervention and prevention. En: Peters RD, Mc Mahon RJ, Quinsey VL, (ed) Agresión and violence through the lifespan. London: Sage Publications 1992: 10025.

12.- Twemlow SW, Fonagy P, Sacco F: The role of bystander in the social architecture of bullying and violence in school and communities. Annals New Cork Academy of Sciences 2004; 1036: 215-32.

13.- Melton GB, Limber S, Cunningham P, Osgood D, Chambers J, Flerx V, Henggeler S, Nation M: Violence among rural youth. Final report to the Office of Juvenile Justice and Delinquency Prevention. 1998.

14.- Charach A, Pepler DJ, Zieler S: Bullying at school: A Canadian perspective. Education Canada 1995; 35: 128.

15.- Rigby K: Psychosocial functioning in families of Australian adolescent schoolchildren envolved in bully/ victim problems. Journal of Family Therapy 1994; 16: $173-87$.

16.- Espelage DL, Bosworth K, Simon TR: Examining the social context of bullying behaviors in early adolescente. Journal of Counseling and Development 2000; 78: 326-33.

17.- Rigby K: How successful are anti-bullying programs forschools? Trabajo presentado ante The Role of Schools in Crime Prevention Conference, Melbourne, Australia, 2002.

18.- Smith JD, Schnider BH, Smith PK, Ananiadou K: The effectivness of whole-school antibullying programs: a síntesis of evaluation research. School Psych Rev 2004; 33: 547-60.

19.- Rigby K: Addressing bullying in schools. Trends Issues Crime Criminal Justice 2003; 259: 1-6.

20.- Vreeman RC, Carrol AE: A systematic review of school-based interventions to prevent bullying. Archives of Pediatrics and Adolescent medicine 2007; 
161: 78-88.

21.- Rigby K, Johnson B: Student bystanders in Australian schools. Pastoral Care 2005; 23: 10-6.

22.- Limber S: Educational Forum on Adolescent Health, Youth bullying. American Medical Association, 2002.

23.- Trautmann A: Victimización entre pares, matonaje o “bullying”. En Vargas N. (ed.). Pediatría diaria para el bicentenario. Patrocinado por Nestle Nutrition, 2007; 303-21.

24.- Glew G, Rivara F, Feudtner C: Bullying: children hurting children. Pediatr Rev 2000; 21: 183-9.

25.- Vessey J, Swanson M, Hagedorn M: Teasing: who says names can never hurt you? Pediatr Nurs 1995; 21: 298-300. 